\title{
Incidental Diagnosis of Carotid Body Paraganglioma
}

\section{Diagnóstico Incidental de Paraganglioma do Corpo Carotídeo}

Silvio Henrique Barberato ${ }^{1}$

${ }^{1}$ CardioEco Cardiovascular Diagnostic Center, Curitiba, PR, Brazil.
Paragangliomas are rare benign neuroendocrine tumors of extra-adrenal location, but a small proportion may have a clinical malignant behavior and metastasize. In the neck, they usually affect the carotid body and are inactive in the secretion of catecholamines. These tumors are often asymptomatic but may present as a pulsatile cervical mass or cause compression in adjacent structures. Surgical excision is the only potentially curative treatment since histological evaluations may be limited to provide a definitive diagnosis of malignancy. Late recurrence can occur. Here we present the case of a 56-year-old asymptomatic woman who underwent carotid ultrasound to screen for atherosclerotic disease in whom a vascularized well-defined hypoechoic mass was incidentally noted in the carotid bifurcation (Figures $1 \mathrm{~A}$ and $1 \mathrm{~B}$ ). Contrast computed tomography confirmed findings compatible with paraganglioma (Figures 1C-1E). The patient underwent surgery and the diagnosis was confirmed.

\section{Conflict of interest}

The author declared that have no conflict of interest.

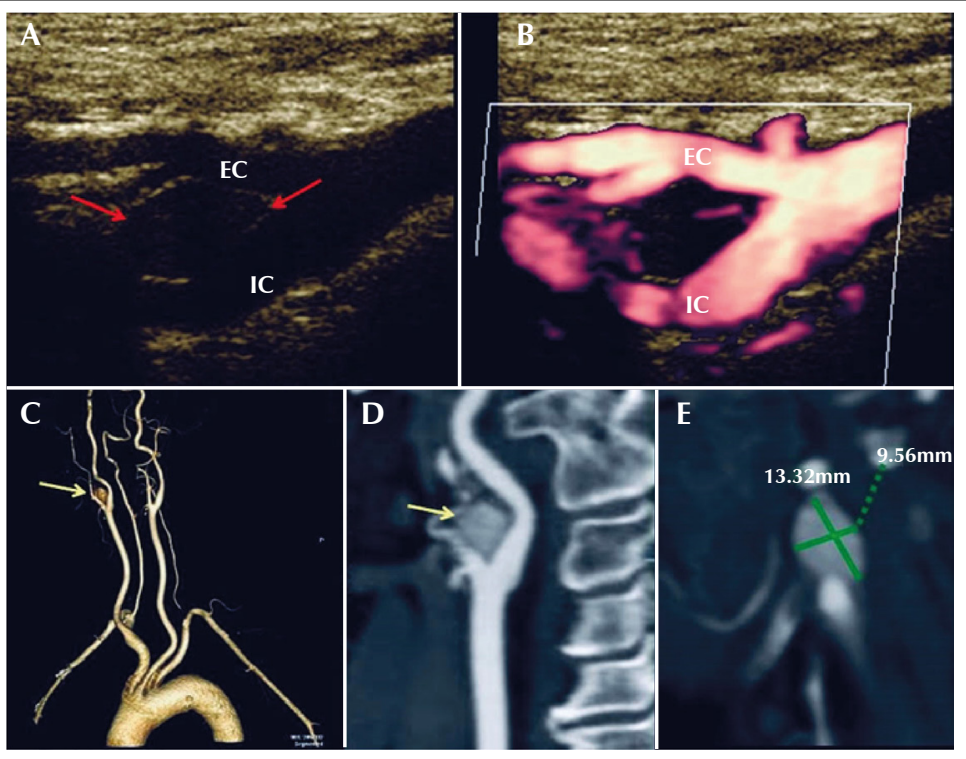

EC, external carotid artery; IC, internal carotid artery.

Figure 1 - (A) Carotid Doppler shows a hypoechoic mass (red arrows) at the carotid bifurcation between the internal and external carotid arteries. (B) Power Doppler imaging enhanced the mass boundaries and vascularity. ( $C$ and D) Contrast computed tomography showing the small mass (yellow arrows) with well-defined limits and homogeneous contrast absorption (E) measuring approximately $13 \times 10 \mathrm{~mm}$.

\section{Keywords}

Ultrasonography, Doppler; Common Carotid Artery; Carotid body tumor; Paraganglioma.

Mailing Address: Silvio Henrique Barberato

Avenida República Argentina, 1.336, sala 215. CEP 80620-010 - Curitiba,

PR, Brazil.

E-mail: silviohb@cardiol.br

Manuscript received 10/13/2021; revised 10/15/2021; accepted 11/3/2021.

DOI: $10.47593 / 2675-312 X / 20213404$ eabc261 